\title{
PPM1H is Down-Regulated by ATF6 and Dephosphorylates p-RPS6KB1 to Inhibit Progression of Hepatocellular Carcinoma
}

\author{
Xiaoshuang Yang \\ Chinese Academy of Medical Sciences and Peking Union Medical College Institute of Basic Medical Sciences \\ Jianting Guo \\ Chinese Academy of Medical Sciences and Peking Union Medical College Institute of Basic Medical Sciences \\ Wei Li \\ The Affiliated Hospital of Qingdao University \\ Chunrui Li \\ Beijing Computing Center \\ Xilin Zhu \\ Chinese Academy of Medical Sciences and Peking Union Medical College Institute of Basic Medical Sciences \\ Ying Liu \\ Chinese Academy of Medical Sciences and Peking Union Medical College Institute of Basic Medical Sciences \\ Xiaopan Wu ( $\nabla$ wuxiaopanpumc@163.com) \\ Chinese Academy of Medical Sciences and Peking Union Medical College Institute of Basic Medical Sciences
}

\section{Research}

Keywords: ATF6, Protein Phosphatase 1H, RPS6KB1, Homology modeling, Hepatocellular carcinoma

Posted Date: August 31st, 2021

DOI: https://doi.org/10.21203/rs.3.rs-817342/v1

License: (c) (i) This work is licensed under a Creative Commons Attribution 4.0 International License. Read Full License 


\section{Abstract}

Background: Our previous studies found that polymorphism of activating transcription factor 6 (ATF6) was associated with susceptibility to hepatocellular carcinoma (HCC), and ATF6 promoted HCC progression. So genes down-regulated by ATF6 might play a tumor suppressing role. In present study, we aimed to identify downstream genes down-regulated by ATF6, and clarified its role in HCC progression.

Methods: To determine the role of ATF6 on downstream gene expression, qRT-PCR and western blot were performed in HCC cells and liver specifically Atf6knocked out mice. Tumor tissues from $134 \mathrm{HCC}$ patients were analyzed by immunohistochemistry. Then, MTT, transwell and clone formation assays were performed in hepatoma cells. DEN/CCl 4 induced $\mathrm{HCC}$ mouse model and nude mice tumorigenicity assay were conducted to investigate the effect of PPM1 $\mathrm{H}$ on $\mathrm{HCC}$ tumorigenesis and development. 3D model of PPM1H was obtained by homology multi-template modeling, the potential substrates of PPM1 H were screened by Rosetta and verified through co-immunoprecipitation, western blot and in vitro phosphatase assays.

Results: The expression of Protein phosphatase magnesium or manganous dependent 1H (PPM1H) mRNA and protein can be inhibited by ATF6. PPM1H inhibited proliferation, migration, invasion and clone formation of cancer cells. PPM1H inhibited induced HCC nodules formation and tumor xenograft growth in two mouse models. The protein docking energy of RPS6KB1 with PPM1H in the BMP/TGF $\beta$ pathway is the lowest, and PPM1H directly dephosphorylated pRPS6KB1. PPM1H exhibited higher expression level in adjacent para-cancer tissues than in HCC tissues. Patients with higher expression of PPM1H indicated a better prognosis.

Conclusions: We first discovered RPS6KB1 as a new PPM1H dephosphorylation substrate. PPM1H exhibited suppressive effect on the progression of HCC through dephosphorylating p-RPS6KB1. This might contribute to finding novel target for therapy of HCC.

\section{Highlights}

1. We first discovered RPS6KB1 as a new PPM1H dephosphorylation substrate

2. ATF6 inhibited the expression of PPM1H at mRNA and protein levels, and promoted proliferation of HCC cells

3. PPM1H inhibited HCC in mouse model and was associated with longer overall survival time in HCC patients.

\section{Background}

Currently, liver cancer is one of the most common malignant tumors, causing about 841,000 new cases and 782,000 deaths in the world every year according to GLOBOCAN in $2018^{1}$. Thus, liver cancer ranks the fifth in terms of global cases, and the second in terms of deaths for males. Among those, $55 \%$ of patients comes from China. Hepatocellular carcinoma (HCC) is the most common types of liver cancer (comprising $75 \%-85 \%$ of cases). Other liver cancer types include intrahepatic cholangiocarcinoma (comprising $10 \%-15 \%$ of cases), as well as other rare types ${ }^{1}$. Due to the lack of reliable early diagnostic markers and effective treatments, clinical treatment for liver cancer is still not satisfactory ${ }^{2,3}$. Therefore, exploring the pathogenesis for HCC and developing markers that related to early diagnosis and prognosis become increasingly important and urgent for finding effective and new treatment for HCC.

Endoplasmic reticulum (ER) is a membrane-bound organelle that synthesizes proteins and lipids in cells ${ }^{4,5}$. Destruction of the ER's homeostasis can result in improper protein folding, processing and transporting. When the redox environment in the ER cavity is destroyed, the accumulation of unfolded or misfolded proteins can cause the endoplasmic reticulum stress (ERS) mechanism. ERS activates the downstream unfolded protein response (UPR) when the stimulating factors persist, which leads to programmed cell death ${ }^{6,7}$. Activated UPR activates downstream protein kinase-like ER kinase (PERK), inositol-requiring enzyme1 (IRE1) and activating transcription factor 6 (ATF6) ${ }^{8-10}$. Accumulated evidence indicates that activating factors induce ERS, which causes ATF6 cleavage, perhaps even to HCC development ${ }^{11}$. Furthermore, our previous studies found that polymorphism of ATF6 was associated with susceptibility to HCC ${ }^{12}$, and ATF6 promoted the progression of $\mathrm{HCC}^{13}$. CTH was identified as a downstream gene of ATF6 through RNA-sequencing (RNA-seq) in HepG2 cells. (SAMN11835389). CTH enhanced the production of $\mathrm{H}_{2} \mathrm{~S}$ in hepatoma cells and promoted HCC development ${ }^{13}$. We also established a mouse model in the previous research. Atf $6^{f / f l}$ C57BL/6 mice possessing loxP sites in Atf6 gene and Alb-cre C57BL/6 mice hybridized and generated liver-specifically Atf6 deficient $\left(A t f 6^{\Delta h e p}\right)$ mice. Considering the tumor-promoting role of ATF6, genes down-regulated by ATF6 might play a tumor suppressing role in HCC development. So in the present study, we aimed to identify downstream genes down-regulated by ATF6, and clarified its role in HCC progression.

\section{Materials And Methods Cell culture}

HepG2, Huh7 and Hep3B cell lines were purchased from the Cell Culture Center of Chinese Academy of Medical Sciences (Beijing, China). They were cultured in Dulbecco's Modified Eagle's Medium and Minimum Essential Medium (Gibco Life Technologies) containing 10\% fetal bovine serum (FBS, Gibco Life Technologies) and maintained at $37^{\circ} \mathrm{C}$ in a moist atmosphere with $5 \% \mathrm{CO}_{2}$.

\section{Animals}

The $A t f \sigma^{f / / f l} \mathrm{C} 57 \mathrm{BL} / 6$ mice were purchased from the Jackson Laboratory (ME, USA). Alb-cre C57BL/6 mice were obtained from the Model Animal Rearch Center of Nanjing University (Nanjing, China). Atf6 $6^{f / f l}$ mice and Alb-cre mice hybridized and generated liver-specifically Atf6 deficient $A t f 6^{\Delta h e p}$ mice. Wild type C57BL/6 mice and BALB/c nude mice were purchased from the Center of Medical Experimental Animals, Chinese Academy of Medical Sciences (Beijing, China). Due to the great gender differences in the incidence of liver cancer, male mice were chosen to perform the experiments in this study. The animal 
feeding and experimental protocols has been approved by the Animal Care and Use Committees and Ethics Committee of the Institute of Basic Medical Sciences, Chinese Academy of Medical Sciences.

\section{Plasmids and transfection}

ATF6, ATF6(1-7), PPM1H and RPS6KB1 coding sequence were inserted into Lv-ef1a-IRES-GFP or p3XFLAG-CMV ${ }^{\mathrm{TM}}$-14 vectors to construct the overexpression plasmids. shATF6 and shPPM1H sequences were inserted into pGPU6/GFP/Neo vectors to establish the knockdown plasmids (Generay Biotechnology, Shanghai, China). PPM1H promoters were inserted into pGL3-basic to construct luciferase reporter plasmids. The target plasmid of interest was transfected into cells using Lipofectamine ${ }^{\text {TM }} 3000$ reagent (Invitrogen, USA).

\section{Antibodies}

Antibodies against ATF6 (polyclonal rabbit, cat no.DF6009), RPS6KB1 (polyclonal rabbit, cat no.AF6226), p-RPS6KB1 (phospho-RPS6KB1, Thr389/Thr412; polyclonal rabbit, cat no.AF3228), Flag-tag (polyclonal rabbit, cat no. T0053) were purchased from Affinity (OH, USA). Antibodies against E-cadherin (monoclonal mouse, cat no. ab231303), N-cadherin (monoclonal rabbit, cat no. ab76011) were purchased from Abcam (Cambridge, UK). Antibodies against pSMAD1 (phospho-SMAD1, Ser463/465; polyclonal rabbit, cat no. 13820T), Flag-tag (polyclonal mouse, cat no. 8146) were purchased from Cell Signaling Technology (MA, USA). Anti-PPM1H (polyclonal rabbit, cat no. CSB-PA892345LA01HU) antibody was purchased from CUSABIO (Wuhan, China).

\section{Human subjects}

134 paraffin-embedded tissues and basic information of HCC patients were collected from the Affiliated Hospital of Qingdao University between 2010 and 2015. All treatment with the tissues had acquired informed consent of the patients.

\section{Stable cell lines establishment and nude mouse tumorigenicity assay}

LV-ATF6, LV-PPM1H, or control plasmids were co-transfected with lentivirus packaging plasmids (delta8.91 and pVSVG) respectively into $293 \mathrm{~T}$ cells planted in 6-well plates. The supernatant culture medium containing corresponding lentivirus were collected $72 \mathrm{~h}$ after transfection and used to infect Hep3B cells planted in 6-well plates. Cells stably expressing ATF6 or PPM1H were selected by EGFP using flow cytometry. By geneticin (G418) selection of Hep3B cell transiently transfected with pGPU6/GFP/Neo-sh-PPM1H plasmid, stable sh-PPM1H cell line was established. $5 \times 10^{6}$ cells were subcutaneously injected into male BALB/c nude mice aged 4 weeks. Xenogeneic tumors were collected 4 weeks later.

\section{$\mathrm{DEN} / \mathrm{CCl}_{4}$-induced $\mathrm{HCC}$ mouse model}

Adeno-associated virus AAV8-PPM1H or AAV8-control were generated by Vigene Bioscience Company (Jinan, China). Wild type or Atf6 ${ }^{\Delta h e p}$ C57BL/6 mice were injected with $25 \mathrm{mg} / \mathrm{kg}$ DEN (Sigma Aldrich, USA) intraperitoneally 2 weeks after birth. Then $5 \mathrm{~mL} / \mathrm{kg} \mathrm{CCl}_{4}$ (Sigma Aldrich, USA) were injected intraperitoneally twice a week for 24 weeks to induce HCC, and $1 \times 10^{12} \mathrm{vg} /$ animal ( $\mathrm{vg}$, viral genome equivalents) AAV8-PPM1H or AAV8-control were injected into mice via tail vein 8 weeks after birth. 3 days after the final injection of $\mathrm{CCl}_{4}$, mice were sacrificed, and livers were extracted.

\section{Dual luciferase assay}

HepG2 cells planted in 24-well plates were co-transfected with PPM1H luciferase reporter plasmids and ATF6 or control plasmids. The luciferase activity was analyzed 48h after transfection using Dual-Luciferase Reporter Assay System (Promega, USA).

\section{Quantitative real-time PCR (qRT-PCR)}

Total RNA of cells or mouse liver tissues were extracted (Ultrapure RNA Kit, CWBIO, Beijing, China) and reverse-transcribed (ReverTra Ace ${ }^{\circledR}$ qPCR RT Master Mix with gDNA Remover, Toyobo life science, Shanghai, China) into cDNA, then qRT-PCR was performed using MagicSYBR Mixture (CWBIO, Beijing, China). Primer sequence was listed in Table. S1.

\section{MTT assay}

$2 \times 10^{3}$ cells planted in 96-well plate were transfected with plasmids or a certain concentration of recombinant protein. $20 \mu \mathrm{L} 3-(4,5-$ dimethylthiazol-2-yl)-2,5diphenyltetrazolium bromide (MTT, Sigma Aldrich, USA) was added. After 4 hours of incubation, OD value at $490 \mathrm{~nm}$ was detected to reflect the proliferation of HCC cells

\section{Transwell migration and invasions}

For cell migration assay, $2 \times 10^{5} \mathrm{HepG} 2$ or Huh7 cells suspended in serum-free medium were directly planted in transwell upper chamber after transfection of target plasmids and the chamber was kept in cell culture medium supplemented with $20 \%$ serum. While for the cell invasion assay, cells were seeded in matrigel basement membrane matrix in transwell chamber. After 48 hours of incubation, cells were fixed with $4 \%$ neutral formaldehyde for 20 minutes, followed by staining with $0.1 \%$ crystal violet for 10 minutes and washing with PBS. The migration and invasion were observed via an inverted microscope. For the RBS6KB1 or SMAD1 blocking, PF-4708671 (MedChemExpress, Shanghai, China) or LDN-193189 (MedChemExpress, Shanghai, China) was added to the culture medium at a final concentration of $10 \mu \mathrm{M}$ and $0.5 \mu \mathrm{M}$ respectively.

\section{Western blot}

Total protein extracted from cells or mouse liver tissues with RIPA lysis buffer (CWBIO, Beijing, China) containing protease inhibitors (phenylmethylsulfonyl fluoride, PMSF; VWR, USA) were separated via sodium dodecyl sulfate polyacrylamide gel electrophoresis (SDS-PAGE) and transferred to polyvinylidene fluoride (PVDF) membranes (Millipore; Billerica, MA, USA). After blocking in 5\% non-fat powdered milk, the membranes were incubated with specific primary 
antibodies at $4{ }^{\circ} \mathrm{C}$ overnight, following HRP-conjugated secondary antibodies incubation at room temperature for 1 hour. Chemiluminescence images were taken by Tanon-5200Multi (Shanghai, China) using Pierce ${ }^{T M}$ ECL western blotting substrate (Thermo Fisher Scientific, Inc., USA).

\section{Co-Immunoprecipitation (Co-IP)}

Total protein dissolved in RIPA lysis buffer (CWBIO, Beijing, China) were incubated with specific antibodies or isotype nonspecific negative control antibodies at $4^{\circ} \mathrm{C}$ overnight with rotation. Protein A/G plus-agarose (Santa Cruz Biotechnology, Inc.CA, USA) was added to incubate for another 2 hours. Then wash the agarose beads with cold PBS for 3 times and centrifugate at $4^{\circ} \mathrm{C}, 3000 \mathrm{rpm}$ for 5 minutes each time to harvest the immunoprecipitated protein.

Immunoblotting was performed to examine the interest proteins. For the in vitro phosphatase assay, the agarose beads were diluted with $20 \%$ glycerol PBS

containing protease inhibitors and preserved in $-80^{\circ} \mathrm{C}$.

\section{Protein purification of prokaryotic expressed PTD-ATF6 and PPM1H}

PTD (protein transduction domain of trans activator of transcription) is a short of peptide (YGRKKRRQRRR) that mediates protein entry into cell ${ }^{14}$, PTD-ATF6 or PPM1H coding sequence were inserted into pET-28a + plasmids, after transferred into E. coli strain Transetta (DE3) (Transgen biotech, China), the recombinant proteins were induced by IPTG, the supernatant was subjected to Ni affinity chromatography after ultrasonic lysis of the bacteria solution (6xHisTagged Protein Purification Kit - Soluble Protein, CWBIO, Beijing, China). Then proteins were concentrated using an ultrafiltration centrifuge tube (Millipore, USA) with a filter pore size of $30 \mathrm{kDa}$. The purified His-PPM1 $\mathrm{H}$ was used to perform in vitro phosphatase assay. PTD-ATF6 was applied to incubate cells at a certain concentration, and the culture medium was changed after 48 hours.

\section{In vitro phosphatase assay}

Immunoprecipitated p-RPS6KB1 was incubated with increasing dosages of PPM1H protein purified from bacteria at $37^{\circ} \mathrm{C}$ for 30 minutes. The mixtures were then subjected to western blot using specific antibodies against p-RPS6KB1 or total RPS6KB1.

\section{PPM1H structural model construction and substrates screening}

The 3D structure of PPM1H was predicted by Modeler software using homologous multi-templates 4jnd, 4ds8, 2isn. Then the model was optimized for molecular dynamics using AMBER14 software through two steps. Firstly, the protein was kept in a free state and to maintain the minimum energy; secondly, the long-range electrostatic energy was calculated using the PME method. The stability of the constructed model was evaluated based on the (skeletal atom rms fluctuation) RMSD value and the Tip3P water box model.

The pathway proteins of PI3K-Akt and BMP/TGF $\beta^{\prime}$ s docking analysis used Rosetta software. The protein in which the PPM1H is stably docked and is screened based on the energy list of the PPM1H prediction model combined with each pathway protein. The spatial combined conformation is further simulated by software.

\section{Immunohistochemistry (IHC)}

Tissue slides of HCC patients and mice were incubated with PPM1H antibody. The immunohistochemistry process was carried out by Servicebio (Wuhan, China).

\section{RNA-sequencing (RNA-seq)}

Total RNA of $A t f 6^{f / f l}$ or $A t f 6^{\Delta h e p}$ C57BL/6 mice liver tissues were used to perform RNA-sEq. The process was completed by Igenecode (Beijing, China). Statistical analysis

Statistical analysis was performed in GraphPad 8.0 software. Each statistical result was determined by three independent experiments. A paired t-test test and Wilcoxon signed rank test were used.

\section{Results}

\section{ATF6 inhibited the expression of PPM1H}

We first tried to identify downstream genes negatively regulated by ATF6. According to RNA-seq data (SAMN11835389) in HepG2 cells ${ }^{13}$, 140 transcripts with significant difference (padj< 0.001) in expression were screened out. To further verify the above results in HepG2 cells, we conducted new RNA-seq in Atf6 ${ }^{f l / f l}$ and $A t f 6^{\Delta h e p}$ mice (PRJNA752835). Western Blot assays revealed that ATF6 expression was eliminated in livers of Atf6 $6^{\Delta h e p}$ mice (Fig. 1A). After a cross-over analysis with the RNA-seq results in cells and mice liver tissues, 107 differentially expressed genes were filtered and 91 of them exhibited same regulation trend in cells and mouse tissues. The top 20 genes with most significant differences were listed in heatmap (Fig. 1B). We chose the top 3 down-regulated genes, i. e. LSS, SLA and PPM1H, to examine their mRNA expression in cells and mice liver tissues. PPM1H showed the most pronounced differentiation in both cells and mice validated by qRT-PCR (Fig. S1A). Western Blot, qRT-PCR and immunohistochemistry assays were performed in mice to further confirm this. Consistently, the expression level of PPM1H mRNA and protein markedly increased in Atf6 ${ }^{\Delta h e p}$ mice (Fig. 1A, C, D). GRP78 and CHOP mRNA expression were notability impaired in Atf $6^{\Delta h e p}$ mice which is consistent with previous studies ${ }^{15}$. HCC cell lines HepG 2 and Huh7 were used to further verify the negative regulation between ATF6 and PPM1H. As expected, the expression level of PPM1H mRNA and protein was obviously impaired in ATF6 overexpressed HepG2 and Huh7 cells. On the contrary, knockdown of ATF6 by shATF6 significantly increased the mRNA and protein expression of PPM1H (Fig. 1E, F), which suggest that ATF6 could suppress the expression of PPM1H. 
We designed a recombinant fusion of ATF6 with PTD (Fig. S1B), which is a cell penetrating peptide ${ }^{14}$. the results for incubated cells with different concentrations of PTD-ATF6 suggested that the ATF6 level in cells after treating with $30 \mu \mathrm{g} / \mathrm{mL}$ PTD-ATF6 recombinant protein was higher than other groups (Fig. S1C). Moreover, incubated HepG2 with $30 \mu \mathrm{g} / \mathrm{mL}$ PTD-ATF6 also promoted the proliferation of cancer cells, which was similar to transfection of FlagATF6 (Fig. 1D). This result indicated that the synthesis ATF6 in vitro could enter cells with PTD and affect the proliferation. In addition, 30 $\mu$ g/mL PTD-ATF6 inhibited the expression of PPM1H in HepG2 (Fig. S1E). Moreover, treatment of PTD-ATF6 could promote the mRNA of GRP78 and CHOP and reduced the expression of PPM1H (Fig. S1F), which further confirmed that ATF6 could inhibit the expression of PPM1H. Taken together, these data determined that the mRNA and protein expression of PPM1H is downregulated by ATF6 both in vitro and in vivo.

To explore how ATF6 inhibited the expression of PPM1H, three PPM1H promoter-PGL3 plasmids containing PPM1H 5'UTR sequences, full-length (pro-full) and truncated (pro-716) promoter sequences were constructed to perform dual luciferase assay in HepG2 cells (Fig. S1G). However, the luciferase activities exhibited no significant differences when ATF6 was over-expressed in both three groups (Fig. S1H), stating that ATF6 inhibited PPM1H expression not through transcriptional ways. Actinomycin D were added to cell culture medium to investigate degradation rate of PPM1H mRNA. However, no significant difference was shown between cells with ATF6 over-expression and the control group (Fig. S1I). These results indicated that ATF6 had almost no influence on the promoter activity or mRNA degradation of PPM1H. Plasmid ATF6(1-7) contained the coding sequence of ATF6 exon 1-7, and expressed a mutant ATF6 protein which can interact with other proteins but cannot bind to DNA. Interestingly, ATF6(1-7) obviously inhibited the mRNA expression of PPM1H (Fig. S1J), indicating that ATF6 might play a suppressing role through the interaction with other proteins, but the mechanism needs to be further determined.

\section{PPM1H mediates proliferation and metastasis of hepatocellular carcinoma cells}

We first conducted MTT assay to evaluated the activity of hepatoma cells, which showed that the proliferation of HepG2 cells significantly decreased when PPM1H was overexpressed, while the cell proliferation was increased when PPM1H was inhibited (Fig. S2A). Besides, PPM1H inhibited the migration and invasion of HepG2 and Huh7 cells (Fig. 2A-B, S2B). While knock-down of PPM1H enhanced the migration and invasion of hepatoma cells (Fig. 2C-D, S2B). In addition, the migration and invasion activity of PPM1H-overexpressed hepatoma cells showed no significant difference when ATF6 was knocked-down or not (Fig. 2A-B, S2B). Consistently, in PPM1H knocked-down cells, co-transfecting ATF6 plasmids barely altered the migration and invasion of HepG2 and Huh7 cells (Fig. 2C-D, S2B), indicating that ATF6 regulated the proliferation and metastasis of hepatoma cells through the function of PPM1H. In general, PPM1H has a suppression effect on the growth of hepatoma cells as a downstream gene of ATF6.

Migration and invasion of epithelial-derived malignant cells is associated with epithelial-mesenchymal transition (EMT). It is manifested by a decrease in the cell adhesion molecule E-cadherin and the mesenchymal marker $\mathrm{N}$-cadherin is increased. Western blot analysis of the EMT markers exhibited that PPM1 $\mathrm{H}$ can promote the E-cadherin expression and inhibit the expression of N-cadherin (Fig. S2C). Conversely, inhibiting PPM1H expression resulted in decreasing Ecadherin and increasing N-cadherin (Figure. S2C). Thus, PPM1H might inhibit the migration and invasion of HCC cells by effecting the EMT characteristics of cancers cells.

PPM1H is a specific phosphatase of SMAD1/5/8 complex ${ }^{16}$. ALK3 (Q233D) mutation of BMPR1A could continuously phosphorylate SMAD1. In this study, HepG2 cells were co-transfected with Flag-SMAD1, BMPR1A(ALK3Q233D) and LV-PPM1H. The results showed that the phosphorylation level of SMAD1 in hepatoma cells was markedly decreased under the influence of PPM1H (Fig. S2D). However, after blocking the phosphorylation of SMAD1 by LDN-193189, overexpressing PPM1H still inhibited the transwell migration and invasion of HepG2 and Huh7 cells (Fig. S2E), reminding that there might be other unknown substrates of PPM1H to regulate HCC cell growth and invasion. So it is necessary to search new substrates of PPM1H.

\section{Computer-simulated protein docking experiments predicted RPS6KB1 as a potential binding substrate for PPM1H}

The crystal structure of the SMAD1 protein and the structure similar to the amino acid sequence of PPM1H were searched in the RCSB PDB database (Table. S2). 4jnd, 4ds8, and 2isn homologous sequences were used as modeling templates (the similarity was $20.26 \%, 31.98 \%$ and $23.66 \%$ respectively). A multitemplate protein modeling method was adopted by Modeler software in order to construct 3D structure prediction of PPM1H protein. Preliminary molecular dynamics optimization was performed by first modeling the PPM1H results. Preliminary molecular dynamics simulation optimization was performed using AMBER14, eliminated the steric hindrance between amino acids in modeling and the unreasonable localized regions of amino acids. A more reliable protein optimized structure was obtained (Fig. 3A). After modeling dynamics optimization, the fluctuation of the RMSD was less than $0.5 \AA$ according to the RMSD of skeleton atoms in the system after 400 ps, indicating the structure of the PPM1H model had reached equilibrium (Fig. 3B). On the other hand, the average node structure of the molecular dynamics trajectory of the target protein in the Tip3P water box model was shown in the computer simulation state, result displayed that the PPM1H model constructed above was structurally stable (Fig. 3C). In our study, we used computer to simulate the biological environment, and used the characteristics of intermolecular interaction and protein stability to predict the spatial structure of PPM1 $\mathrm{H}$, especially its active region.

We screened potential substrates for PPM1H through computer simulation according to the structural characteristics for PPM1H, it mainly includes substrates with serine/threonine in active center for activation ${ }^{17-19}$. On the other hand, we hypothesized that there were potential substrates of PPM1 H in PI3K/Akt and BMP/TGF $\beta$ pathways, which could affect the progression of HCC.

The potential substrates for PPM1H in the PI3K/Akt and BMP/TGF $\beta$ pathways were screened by computer-simulated protein docking experiments. All protein information in both pathways was obtained from the KEGG Pathway database, and then downloaded the RCSB PDB structure information for pathway protein. The Rosetta software was used to analysis the 113 proteins contained in the pathways, and the protein-protein molecules were docked separately. The closest clustering of nearly 10,000 protein docking phases was generated, and top 10 of energy of PPM1H docking conformations was shown in Table. 1 . The best energy-matching docking phase conformation: $-26.51 \mathrm{Kcal} / \mathrm{moL}$ as the optimal docking result of the system (Fig. 3D). Table. 1 was the energy of docking conformation, then the optimal conformation of the PPM1H were docked with the total of 105 protein targets in the PI3K/Akt and BMP/ TGF $\beta$ pathway. 
According to the docked energy of proteins with best conformation for PPM1H, ElF4B (-25.21 Kcal/moL) and RPS6KB1 (-25.31 Kcal/moL) respectively were the most stable protein target in each signaling pathways. Figure 3E shows the three-dimensional docking structure diagram and binding interface of PPM1H and EIF4B, RPS6KB1, and the binding sites of PPM1H with two predicted substrates was located at the enzyme activity center. Meanwhile, the docking energy of SMAD1 with PPM1H was $-19.21 \mathrm{Kcal} / \mathrm{moL}$, second only to RPS6KB1 in BMP/TGF $\beta$ pathway. Finally, according to the structure of PPM1 $\mathrm{H}$ simulated by computer and the analysis of the docking energy between PPM1H and potential substrates, we found 16 substrates from PI3K/Akt and BMP/TGF $\beta$ with lower docking energy than SMAD1, among which EIF4B and RPS6KB1 have the lowest docking energy and the most stable binding energy with PPM1H.

\section{PPM1H directly dephosphorylated p-RPS6KB1}

The docking energy of RPS6KB1 with PPM1H was $-25.31 \mathrm{Kcal} / \mathrm{moL}$, lower than SMAD1 in BMP/TGF $\beta$ pathway, indicating it a promising substrate of PPM1H. To determine if PPM1H interacted with RPS6KB1, co-immunoprecipitation (Co-IP) were conducted in HepG2 and Huh7 cells with insulin (10ug/mL) served as a mTOR/RPS6KB1 activator. PPM1H could indeed interact with RPS6KB1, and the interaction between them were significantly enhanced upon insulin added (Fig. 4A-B, S3A-B). Next, we confirmed that p-RPS6KB1 markedly reduced in PPM1H overexpressed HepG2 and Huh7 cells (Fig. 4C, S3C). Instead, knocked-down of PPM1H increased the level of p-RPS6KB1 (Fig. S3D). To further determined the direct dephosphorylation of PPM1H to p-RPS6KB1 in vitro, immunoprecipitated RPS6KB1 was incubated with increasing dose of recombinant His-PPM1H protein extracted from bacteria. As a result, PPM1H eliminated p-RPS6KB1 in a dose-dependent manner (Fig. 4D). The expression level of PPM1H was negatively regulated by ATF6 according to our previous research. In ATF6 overexpressed HepG2 and Huh7 cells, phosphorylation level of RPS6KB1 significantly increased, and knock-down of ATF6 inhibited the pRPS6KB1 expression (Fig. 4E, S3E). The liver tissues of $A t f 6^{\Delta h e p}$ mice also have a lower expression level of p-RPS6KB1 than the Atf $f^{f / f l}$ mice (Fig. 4F), which suggested that ATF6 promoted the phosphorylation of RPS6KB1 through the inhibition of PPM1H. RPS6KB1 inhibitor PF-4708671 were used to verify if PPM1H affected the HCC progression through RPS6KB1. The transwell migration and invasion assays showed that there was little difference between PPM1H-overexpressing and control groups after PF-4708671 were added (Fig. 4G), proving that PPM1H regulate the growth of hepatoma cells through the inhibition of RPS6KB1.

\section{PPM1H suppressing tumor growth in mouse HCC models}

To determine if PPM1H inhibit tumor growth in vivo, we established stable PPM1H or ATF6 overexpression and sh-PPM1H Hep3B cells. The stable cell lines were then subcutaneously injected into male BALB/c nude mouse. As shown in Fig. 5A, knock down of PPM1H obviously enhance the tumorigenicity of Hep3B cells, while PPM1H-overexressing Hep3B cells formed smaller xenogeneic tumor than the control group. Besides, ATF6 promoted the tumorigenicity of Hep3B cells, and this could be partially neutralized after ATF6-overexressed Hep3B cells were co-infected with PPM1H lentivirus. Among PPM1H overexpressed cells, xenogeneic tumor size of cells co-infected with ATF6 was a little bit lager than the cells co-infected with control lentivirus (Fig. 5A). These results demonstrated that PPM1H reduced the tumorigenicity of hepatoma cells.

$\mathrm{DEN} / \mathrm{CCl}_{4}$-induced $\mathrm{HCC}$ mouse model was constructed to further investigate the influence of PPM1H on HCC tumorigenesis. As exhibited in Fig. 5B, Wild type or $A t f 6^{\Delta h e p}$ C57BL/ 6 mice were injected with DEN and $\mathrm{CCl}_{4}$ to induce $\mathrm{HCC}$, and Adeno-associated virus AAV8-PPM1H or AAV8-control virus were injected into mice tail vein 8 weeks after birth. After AAV8-PPM1H overexpression, PPM1H was overexpressed and the phosphorylation level of RPS6KB1 was reduce, which were confirmed through western blot analysis (Fig. $5 \mathrm{C}$ ). The expression of cyclin A2 and cyclin B1 can be activated by RPS6KB1 ${ }^{20}$. In the livers of PPM1H overexpressed mice, the mRNA expression of these two downstream genes were also down-regulated (Fig. 5D). In the induced HCC mouse models, there were less tumor nodules in the livers of $P p m 1 \mathrm{~h}$-mice than the control group (Fig. 5E, 5F), and the ratio of liver weight to body weight also significantly reduced in Ppm1 1 -mice (Fig. 5G). The induced HCC mouse model was also constructed in AtfG $\sigma^{f / / f l}$ and Atf ${ }^{\Delta h e p}$ mice. Among the Atf $\sigma^{\Delta h e p}$ mice, PPM1H$A t f 6^{\Delta h e p}$ mice apparently reduced the induced tumor nodules formation and the ratio of liver weight to body weight than the NC-Atf $\sigma^{\Delta h e p}$ mice, while little difference was showed between the PPM1H-Atf6 $6^{f / f l}$ and the PPM1H-Atf6 ${ }^{\Delta h e p}$ mice (Fig. 5H-J), demonstrating that ATF6 promoting the progression of HCC through the regulation of PPM1H. These results revealed that PPM1 H played an inhibitory role in the regulation of HCC tumorigenesis and tumor growth

\section{PPM1H predicted the progression of HCC patients}

To verify the PPM1H expression level of different cancer types, we analyzed breast cancer (BRCA), cholangiocarcinoma (CHOL), lung adenocarcinoma (LUAD) and liver cancer (LIHC) disclosed in the cancer genome atlas (TCGA) database (https://www.cancer.gov/tcga). The results showed that PPM1H expression in cancer tissues was higher than that in normal tissues in BRCA $(n=1085)$, CHOL $(n=36)$ and LUAD $(n=483)$. PPM1H was lowly expressed in LIHC ( $=369)$ tumor tissues, although there were no significant differences (Fig. S4A). In this study, we used immunohistochemistry to detect the expression of PPM1H in 134 paraffin-embedded liver cancer tissues. The results showed that PPM1H was expressed in both cancer and adjacent para-cancer tissues, the overall staining intensity of PPM1H in tumor tissues is lower than the adjacent tissues, which is in consistent with the trend in TCGA database, while the number of nuclear staining positive cells in tumor tissues was more than that in adjacent tissues, hint that the PPM1H expression might be complementally increased in nuclei to exert anticancer functions (Fig. 6A). But this speculation needed to be further verified. Finally, survival analysis was conducted. Patients were divided into two groups according to PPM1H expression levels in HCC tissues (Fig. 6B), and the overall survival time of patients with high PPM1H expression level was obviously longer than patients expressing lower level of PPM1H (Fig. 6C), reminding that PPM1H might predicted the prognosis of HCC patients.

\section{Discussion}

In this study we demonstrated that ATF6 inhibited the PPM1H mRNA and protein expression. PPM1H decreased the phosphorylation level of PRS6KB1. The proliferation, migration, invasion and tumorigenicity of hepatoma cells and the induced mouse HCC were suppressed by PPM1H (Fig. 7).

The serine/threonine phosphatase PPM1H is determined as a new family member of the PPM family which is expressed in both cytoplasm and nucleus. The similarity of PPM1H to PPM1J (also known as PP2Ç) in the phosphatase active region is as high as $80 \%{ }^{21}$, which also suggests that its enzyme activity 
region might be similar to other family members. PPM1H was reported to be related to cell proliferation, differentiation, and tumor progression ${ }^{16,22}$. However, the role of PPM1H may differ depending on the cancer type. It was downregulated in glioblastoma while upregulated in colon cancer ${ }^{22,23}$. These studies suggested that its exact mechanism of action in cancer remains controversial. In our research, we identified the suppression role of PPM1H on hepatoma cell growth, invasion and HCC progression. In addition, PPM1H was downregulated in human HCC tissues and its low expression predicted poor prognosis of HCC patients.

Ribosomal protein S6 kinase B1 (RPS6KB1, also known as p70S6K or S6K1), is a serine/threonine kinase which can be activated by the PI3K/mTOR signaling. RPS6KB1 plays an important role in regulating glucose metabolism ${ }^{24}$, autophagy ${ }^{25}$, cell proliferation and survival ${ }^{26}$. Recent studies find that p-RPS6KB1 is upregulated in prostate cancer and plays essential role in sensitization of radiation therapy ${ }^{27}$. In brain tumors, correlated RPS6KB1 and hypoxia responsive genes are upregulated and RPS6KB1 is associated with poor prognosis of patients ${ }^{28}$. In breast cancer, RPS6KB1 phosphorylates and activates estrogen receptor-a (ERa), which in turn enhances the transcription of RPS6KB1, leading to proliferation of breast cancer cells ${ }^{29}$. RPS6KB1 is also upregulated in human $\mathrm{HCC}$ tissues than the adjacent noncancerous tissues and predicts poor prognosis of HCC patients ${ }^{30}$. In our study, p-RPS6KB1 can be directly dephosphorylated by PPM1H in hepatoma cells. The phosphorylation of RPS6KB1 activated the cell cycle regulatory proteins cyclin A2 and cyclin B1, which then promotes the progression of the hepatocellular carcinoma. This reminds that PPM1H might suppress HCC development through the negative regulation of RPS6KB1 on the cell cycle modulation. Besides, insulin was added to cells to simulate a state of highly activated p-RPS6KB1, an enhanced interaction between PPM1H and RPS6KB1 was observed after insulin treatment, implying that PPM1H play a more effective role under conditions that the RPS6KB1 pathway is highly activated.

The phosphatase activity of PPM1H inhibits the transduction of signaling pathways involved in cancer progression. The recombinant PPM1H can directly act on the SMAD active domain and dephosphorylate the phosphorylated SMAD1/5/8 ${ }^{16}$. In this study, PPM1H inhibited the phosphorylation of SMAD1 and RPS6KB1 in HCC cells. Surprisingly, a suppression effect of PPM1H still remained after the SMAD1 signaling was blocked, while RPS6KB1 blocking greatly impaired the influence of PPM1H on cell migration and invasion. This suggested that RPS6KB1 played a more important role in the modulation of cell growth and invasion under PPM1H regulation.

Protein-protein docking prediction by computational methods is an important complement to the experimental detection of complex structures. Virtual screening based on target structure is used in protein-small molecule docking has been widely used in the screen of small molecule drugs. The active ingredients and ligands are predicted by kinetic evaluation of drug similarity and drug action of substrates, finally provides a scientific basis for exploring the mechanism of treatment of liver cancer ${ }^{31-33}$. In this study, we have successfully verified the effect of PPM1H on p-RPS6KB1 both in vivo and in vitro. However, the model still have a certain gap with the real state, due to the network of protein interactions is extremely complex, more candidates of PPM1 $\mathrm{H}$ may not have been filtered out. In this study, only the one-on-one combination of PPM1H and potential substrates is focused. The computer simulation experiment is used as a prediction for exploring phosphorylation substrates. At present, the influence of PPM1 $\mathrm{H}$ on the other candidate substrates except for RPS6KB1, like EIF4B, has to be further explored, we will continue to verify the candidate substrates in order to find new targets for PPM1H in liver cancer.

Overexpression of ATF6 exhibited little impact on the promoter activity or the mRNA degradation of PPM1H, hint that ATF6 might influence the PPM1H expression through some other ways like enhancers which hasn't been found in this study. Follow-up studies such as ChIP-sequencing will be carried out to unearth the regulation mechanism between ATF6 and PPM1H.

Immunohistochemical analysis of PPM1H expression in liver tissues of HCC patients shows that PPM1H staining intensity in tumor tissues is somewhat lower than that in adjacent non-tumor tissues, which is consistent with the TCGA database analysis. However, the TCGA analysis also shows that PPM1H expression is higher in tumor of BRCA, CHOL, LUAD than in adjacent tissues. In view of the widely distributed sample coverage population, no significant difference was shown in liver cancer tissues. In future studies, we should expand sample size of HCC patients.

\section{Conclusion}

In this study, we determined that the mRNA and protein expression of PPM1H was inhibited by ATF6. PPM1H suppressed growth, invasion and tumorigenicity of hepatoma cells. In HCC patients, low expression of PPM1H was associated with poor prognosis. Besides, we first discovered RPS6KB1 as a new PPM1H dephosphorylation substrate, which might play an important role in the regulation of hepatoma cells development. To some extent, this study revealed the molecular mechanism of HCC development and the ATF6/PPM1H/RPS6KB1 regulating axis provided more proof to find therapeutic targets for HCC.

\section{Abbreviations}

HCC hepatocellular carcinoma

ATF6 activating transcription factor 6

PPM1H Protein phosphatase magnesium or manganous dependent $1 \mathrm{H}$

RPS6KB1 Ribosomal protein S6 kinase B1

EIF4B Eukaryotic initiation factor 4B

CHOP C/EBP homology protein CEBP

GRP78 Glucose-regulated protein78 
TGF- $\beta$ Transforming growth factor $\beta$

BMP Bone morphogenetic protein

EMT Epithelial-mesenchymal transition

NC Negative control

shRNA Short hairpin RNA

RNA-seq RNA-sequencing

qRT-PCR Quantitative real-time PCR

Co-IP Co-immunoprecipitation

IHC Immunohistochemistry

\section{Declarations}

\section{Ethics approval and consent to participate}

All procedures related to human subjects were performed with informed consent and approved by the ethics committee of the Affiliated Hospital of Qingdao University. The experiments related to animals has been approved by the Animal Care and Use Committees and Ethics Committee of the Institute of Basic Medical Sciences, Chinese Academy of Medical Sciences.

\section{Consent for publication}

All authors agree to submit the manuscript for submission.

\section{Availability of data and materials}

The datasets of the current study are available from the corresponding author on reasonable request.

\section{Competing interests}

The authors declare no competing interests.

\section{Funding}

This work was supported by grants from the Chinese Academy of Medical Sciences (CAMS) Innovation Fund for Medical Sciences [2018-I2M-1-004, 2016-I2M$1-004]$.

\section{Authors' contributions}

XPW and YL designed the study. XSY, JTG, CRL and XLZ conducted the experiments and data analysis. XSY, JTG wrote the paper. WL provided subjects from HCC patients. All authors read and approved the final manuscript.

\section{Acknowledgements}

We are thankful to all subjects who participated in this study.

\section{Authors' information}

Xiaoshuang Yang, yangxs0927@163.com

Jianting Guo,gjt0427@163.com

Ying Liu, liuyingpumc@163.com

Xiaopan Wu, wuxiaopanpumc@163.com

\section{References}

1. Bray, F. et al. Global cancer statistics 2018: GLOBOCAN estimates of incidence and mortality worldwide for 36 cancers in 185 countries. CA Cancer J Clin 68, 394-424, doi:10.3322/caac.21492 (2018).

2. Jemal, A. et al. Global cancer statistics. CA Cancer J Clin 61, 69-90, doi:10.3322/caac.20107 (2011).

3. Wong, C. M. \& Ng, I. O. Molecular pathogenesis of hepatocellular carcinoma. Liver Int 28, 160-174, doi:10.1111/j.1478-3231.2007.01637.x (2008). 
4. Diehl, J. A., Fuchs, S. Y. \& Koumenis, C. The cell biology of the unfolded protein response. Gastroenterology 141, 38-41, 41 e31-32, doi:10.1053/j.gastro.2011.05.018 (2011).

5. Hetz, C. The unfolded protein response: controlling cell fate decisions under ER stress and beyond. Nat Rev Mol Cell Bio/ 13, 89-102, doi:10.1038/nrm3270 (2012).

6. Lee, J. \& Ozcan, U. Unfolded protein response signaling and metabolic diseases. J Biol Chem 289, 1203-1211, doi:10.1074/jbc.R113.534743 (2014).

7. Malhi, H. \& Kaufman, R. J. Endoplasmic reticulum stress in liver disease. J Hepato/ 54, 795-809, doi:10.1016/j.jhep.2010.11.005 (2011).

8. Kim, J. O., Kwon, E. J., Song, D. W., Lee, J. S. \& Kim, D. H. miR-185 inhibits endoplasmic reticulum stress-induced apoptosis by targeting Na+/H+ exchanger-1 in the heart. BMB Rep 49, 208-213, doi:10.5483/bmbrep.2016.49.4.193 (2016).

9. Tampakakis, E. et al. Intravenous Lipid Infusion Induces Endoplasmic Reticulum Stress in Endothelial Cells and Blood Mononuclear Cells of Healthy Adults. J Am Heart Assoc 5, doi:10.1161/JAHA.115.002574 (2016).

10. Wang, M. \& Kaufman, R. J. The impact of the endoplasmic reticulum protein-folding environment on cancer development. Nat Rev Cancer 14, 581-597, doi:10.1038/nrc3800 (2014)

11. Li, B. et al. Hepatitis B virus X protein (HBX) activates ATF6 and IRE1-XBP1 pathways of unfolded protein response. Virus Res 124, 44-49, doi:10.1016/j.virusres.2006.09.011 (2007).

12. Wu, X. et al. A missense polymorphism in ATF6 gene is associated with susceptibility to hepatocellular carcinoma probably by altering ATF6 level. Int $J$ Cancer 135, 61-68, doi:10.1002/ijc.28649 (2014).

13. Man Y. Molecular mechanism of ATF6 on regulating downstream gene CTH to affect hepatocellular carcinoma progression. Dissertation, Institute of Basic Medica Sciences, Chinese Academy of Medical Sciences; School of Basic Medicine, Peking Union Medical College, doi: 10.27648/d.cnki.gzxhu.2020.000356. https://kns.cnki.net/kcms/detail/detail.aspx? $\mathrm{dbcode}=$ CDFD\&dbname=CDFDLAST2021\&filename=1021026232.nh\&v=VoudYD95xWI6jpmYz62VH652iOVRSCLn46VOMmvt\%25mmd2FQ6NAXIHmXYK (2020).

14. Chu, X. et al. PTD-fused p53 as a potential antiviral agent directly suppresses HBV transcription and expression. Antiviral Res 127, 41-49, doi:10.1016/j.antiviral.2016.01.008 (2016)

15. Cubillos-Ruiz, J. R., Bettigole, S. E. \& Glimcher, L. H. Tumorigenic and Immunosuppressive Effects of Endoplasmic Reticulum Stress in Cancer. Cel/ 168, 692-706, doi:10.1016/j.cell.2016.12.004 (2017).

16. Shen, T. et al. Specific control of BMP signaling and mesenchymal differentiation by cytoplasmic phosphatase PPM1H. Cell Res $24,727-741$ doi:10.1038/cr.2014.48 (2014).

17. Barr, A. J. et al. Large-scale structural analysis of the classical human protein tyrosine phosphatome. Cell 136, 352-363, doi:10.1016/j.cell.2008.11.038 (2009)

18. Parsons, M., Worthey, E. A., Ward, P. N. \& Mottram, J. C. Comparative analysis of the kinomes of three pathogenic trypanosomatids: Leishmania major, Trypanosoma brucei and Trypanosoma cruzi. BMC Genomics 6, 127, doi:10.1186/1471-2164-6-127 (2005).

19. Shi, Y. Serine/threonine phosphatases: mechanism through structure. Cel/ 139, 468-484, doi:10.1016/j.cell.2009.10.006 (2009).

20. Morzyglod, L. et al. Growth factor receptor binding protein 14 inhibition triggers insulin-induced mouse hepatocyte proliferation and is associated with hepatocellular carcinoma. Hepatology 65, 1352-1368, doi:10.1002/hep.28972 (2017).

21. Sugiura, T. \& Noguchi, Y. Substrate-dependent metal preference of PPM1H, a cancer-associated protein phosphatase 2C: comparison with other family members. Biometals 22, 469-477, doi:10.1007/s10534-009-9204-9 (2009).

22. Zhu, H., Qin, H., Li, D. M., Liu, J. \& Zhao, Q. Effect of PPM1H on malignant phenotype of human pancreatic cancer cells. Oncol Rep 36, 2926-2934, doi:10.3892/or.2016.5065 (2016).

23. Aceto, N. \& Bentires-Alj, M. On the road to combinations of targeted therapies: PPM1H phosphatase as a suppressor of trastuzumab resistance. Cancer Discov 1, 285-286, doi:10.1158/2159-8290.CD-11-0185 (2011)

24. Pende, M. et al. Hypoinsulinaemia, glucose intolerance and diminished beta-cell size in S6K1-deficient mice. Nature 408, 994-997, doi:10.1038/35050135 (2000).

25. Devis-Jauregui, L., Eritja, N., Davis, M. L., Matias-Guiu, X. \& Llobet-Navas, D. Autophagy in the physiological endometrium and cancer. Autophagy 17, 10771095, doi:10.1080/15548627.2020.1752548 (2021).

26. Chen, B. et al. Hyperphosphorylation of RPS6KB1, rather than overexpression, predicts worse prognosis in non-small cell lung cancer patients. PLoS One 12, e0182891, doi:10.1371/journal.pone.0182891 (2017).

27. Hussain, S. S. et al. Suppression of ribosomal protein RPS6KB1 by Nexrutine increases sensitivity of prostate tumors to radiation. Cancer Lett 433, 232241, doi:10.1016/j.canlet.2018.07.009 (2018).

28. Ismail, H. M. Overexpression of s6 kinase 1 in brain tumours is associated with induction of hypoxia-responsive genes and predicts patients' survival. $J$ Oncol 2012, 416927, doi:10.1155/2012/416927 (2012).

29. Maruani, D. M. et al. Estrogenic regulation of S6K1 expression creates a positive regulatory loop in control of breast cancer cell proliferation. Oncogene 31, 5073-5080, doi:10.1038/onc.2011.657 (2012).

30. Li, P. D. et al. Overexpression of RPS6KB1 predicts worse prognosis in primary HCC patients. Med Oncol 29, 3070-3076, doi:10.1007/s12032-012-0268-y (2012).

31. Lokhande, K. B., Nagar, S. \& Swamy, K. V. Molecular interaction studies of Deguelin and its derivatives with Cyclin D1 and Cyclin E in cancer cell signaling pathway: The computational approach. Sci Rep 9, 1778, doi:10.1038/s41598-018-38332-6 (2019).

Page 9/18 
32. Xie, C. et al. Identification of a New Potent Inhibitor Targeting KRAS in Non-small Cell Lung Cancer Cells. Front Pharmaco/ 8, 823, doi:10.3389/fphar.2017.00823 (2017).

33. Zhang, C., Tang, B., Wang, Q. \& Lai, L. Discovery of binding proteins for a protein target using protein-protein docking-based virtual screening. Proteins 82 , 2472-2482, doi:10.1002/prot.24611 (2014).

\section{Tables}


Table 1

Docking energy between PPM1H with PI3K/Akt and BMP/TGF $\beta$ pathway protein

\begin{tabular}{|c|c|c|c|c|c|c|}
\hline \multicolumn{2}{|l|}{ Pathway } & GENE & \multicolumn{2}{|l|}{$\begin{array}{l}\text { Energy } \\
\text { (Kcal/moL) }\end{array}$} & GENE & \multirow{2}{*}{$\begin{array}{l}\text { Energy } \\
\text { (Kcal/moL) } \\
-15.73\end{array}$} \\
\hline PPM1H- & 1 & EIF4B & -25.21 & 25 & GRB2 & \\
\hline \multirow[t]{23}{*}{ PI3K/Akt } & 2 & PIK3AP1 & -24.55 & 26 & MLST8 & -15.36 \\
\hline & 3 & CD19 & -24.44 & 27 & CCND1 & -15.02 \\
\hline & 4 & EIF4EBP1 & -24.30 & 28 & GSK3B & -14.88 \\
\hline & 5 & TSC1 & -23.89 & 29 & BCL2 & -14.59 \\
\hline & 6 & MDM2 & -23.51 & 30 & JAK1 & -14.49 \\
\hline & 7 & HRAS & -23.22 & 31 & CDC37 & -14.40 \\
\hline & 8 & STK11 & -23.14 & 32 & MTOR & -14.22 \\
\hline & 9 & NFKB1 & -23.08 & 33 & GATA1 & -14.09 \\
\hline & 10 & DDIT4 & -23.01 & 34 & CDKN1A & -13.97 \\
\hline & 11 & RPS6 & -22.01 & 35 & PTEN & -13.97 \\
\hline & 12 & RAC-1 & -21.49 & 36 & PDPK1 & -13.67 \\
\hline & 13 & HSP90AA1 & -21.08 & 37 & CSF1 & -13.38 \\
\hline & 14 & CSF1R & -19.86 & 38 & PPP2R3B & -13.09 \\
\hline & 15 & RHEB & -19.77 & 39 & AKT3 & -13.04 \\
\hline & 16 & RPS6KB1 & -18.45 & 40 & CD79A & -12.99 \\
\hline & 17 & RAF1 & -17.25 & 41 & THEM4 & -12.55 \\
\hline & 18 & BRCA1 & -17.17 & 42 & YWHAQ & -12.29 \\
\hline & 19 & CSF3R & -17.09 & 43 & SYK & -12.01 \\
\hline & 20 & F0X03 & -17.08 & 44 & PIK3CA & -11.37 \\
\hline & 21 & PRKAA1 & -17.03 & 45 & CDKN1B & -11.33 \\
\hline & 22 & MTCP1 & -16.30 & 46 & EIF4E & -10.33 \\
\hline & 23 & ITGB1 & -16.22 & 47 & MYC & -10.11 \\
\hline & 24 & CHUK & -16.03 & 48 & & \\
\hline \multirow{14}{*}{$\begin{array}{l}\text { PPM1H- } \\
\text { BMP/TGF } \beta\end{array}$} & 1 & RPS6KB1 & -25.31 & 15 & CREBBP & -13.76 \\
\hline & 2 & SMAD1 & -19.21 & 16 & SMAD2 & -12.98 \\
\hline & 3 & SMAD4 & -17.00 & 17 & SYM1 & -12.73 \\
\hline & 4 & BMP2 & -16.63 & 18 & HJV & -12.54 \\
\hline & 5 & ACVR2A & -16.40 & 19 & SMURF1 & -12.44 \\
\hline & 6 & PITX2 & -15.75 & 20 & BMPR1A & -12.03 \\
\hline & 7 & TGFBR2 & -15.54 & 21 & ZFYVE9 & -11.77 \\
\hline & 8 & IFNG & -15.21 & 22 & THBS1 & -11.38 \\
\hline & 9 & ROCK1 & -14.85 & 23 & SP1 & -10.87 \\
\hline & 10 & HAMP & -14.31 & 24 & E2F-4 & -10.58 \\
\hline & 11 & RBX1 & -14.31 & 25 & MAPK1 & -10.53 \\
\hline & 12 & TNFA & -14.07 & 26 & INHBA & -10.03 \\
\hline & 13 & RBL1 & -13.82 & 27 & BMPR2 & -10.03 \\
\hline & 14 & CUL1 & -13.82 & & & \\
\hline
\end{tabular}

Figures

Page 11/18 
C

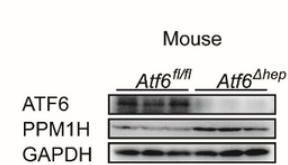

B

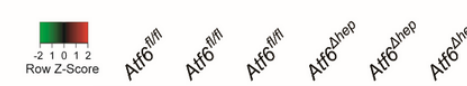

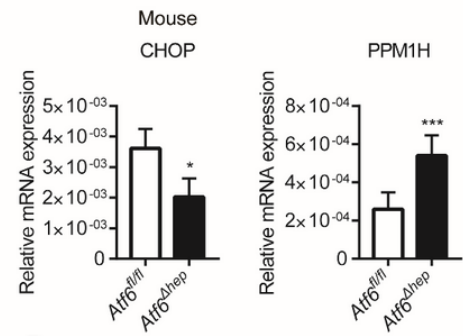

D

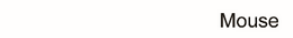

PPM1H immunohistochemical

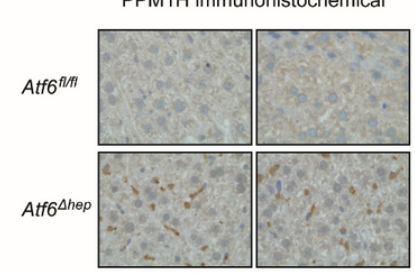

E

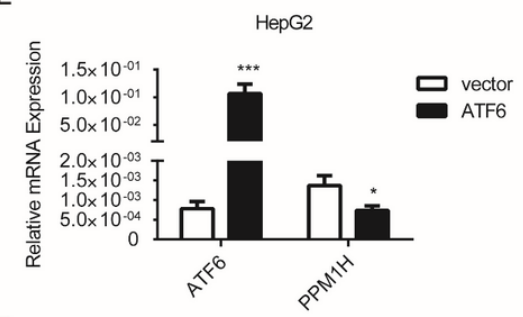

F
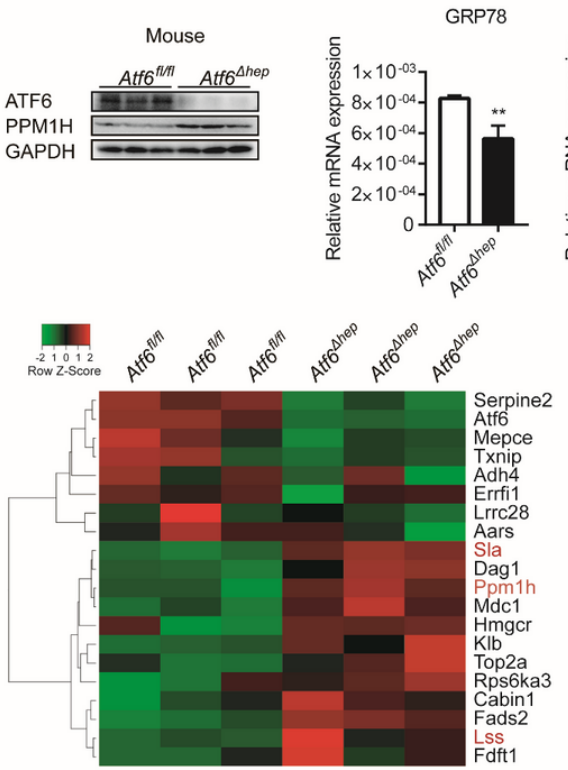

(n)

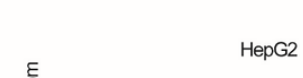

HepG2
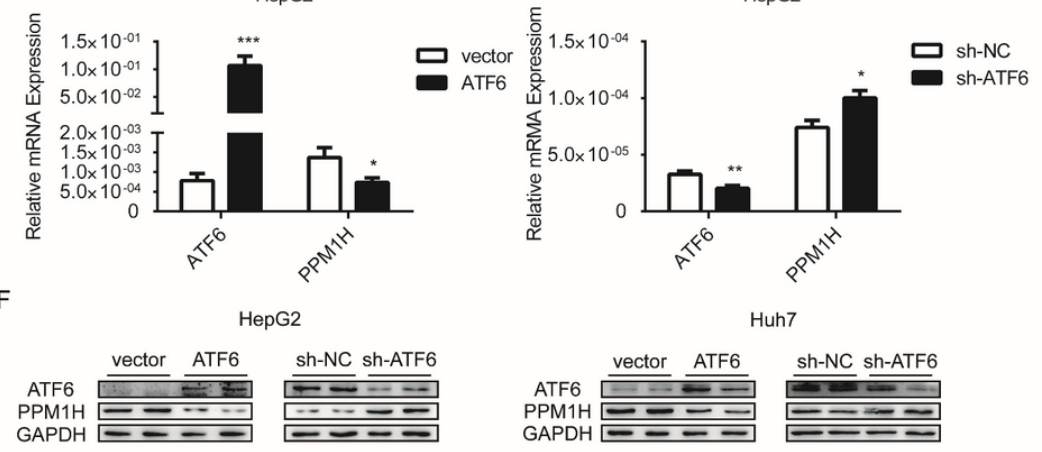

HepG2

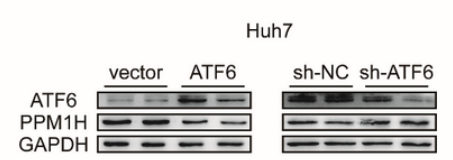

\section{Figure 1}

ATF6 inhibits the expression of PPM1H in hepatoma cells. A: Western blot analysis of the ATF6 and PPM1H protein expression in mouse liver tissues. B: Heatmap of the differentially expressed genes filtered out according to the RNA-seq in HepG2 and mice. C: qRT-PCR analyzed the mRNA expression of GRP78, CHOP and PPM1H in mouse liver tissues. D: IHC assay of PPM1H in the liver tissues of Atf6fl/fl and Atf6 $\Delta$ hep mice. E and F: vector, ATF6, sh-NC or sh-ATF6 plasmids were transfected into HepG2 or Huh7 cells to overexpress or knockdown ATF6, E: qRT-PCR analyzed the mRNA level of ATF6 and PPM1H in HepG2 cells, data was normalized to GAPDH. F: Western blot analysis of the ATF6 and PPM1H protein expression in HepG2 and Huh7 cells. Data represent the mean \pm SD of three independent experiments. ${ }^{*} p<0.05,{ }^{* \star} p<0.01$, ${ }^{* *} p<0.001$. 
A

\section{vector}

PPM1H

PPM1H+sh-NC

PPM1H+ShATF6
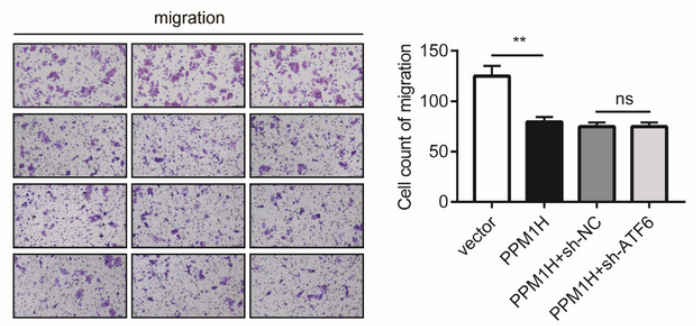

B

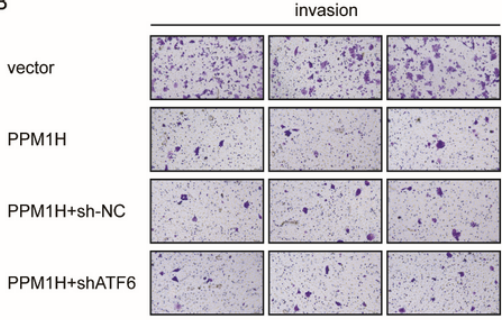

C

sh-NC

sh-PPM1H

sh-PPM1H+vecot

sh-PPM1H+ATF6

D

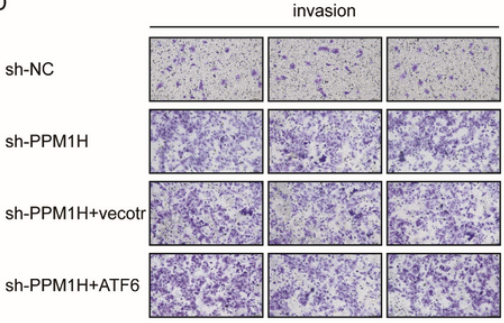

migration
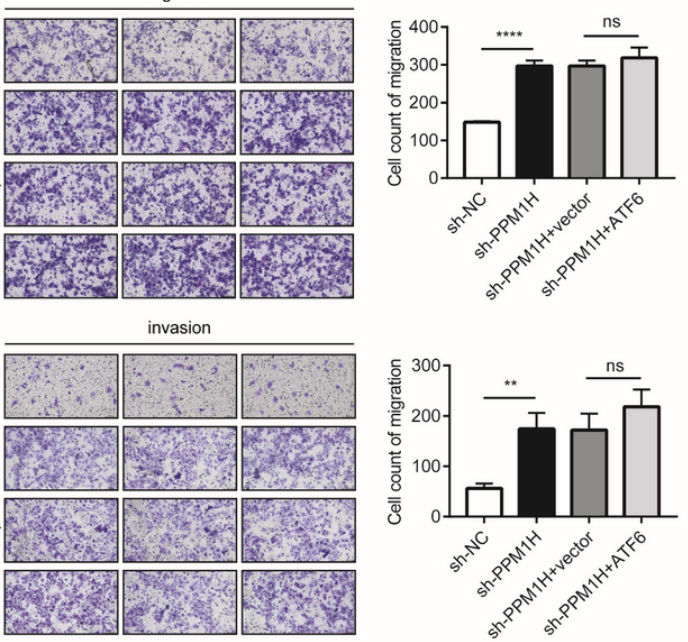

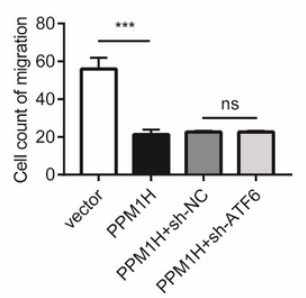

\section{Figure 2}

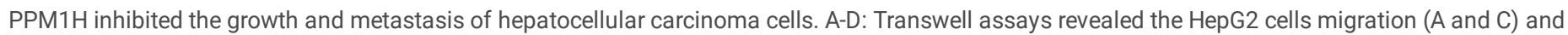

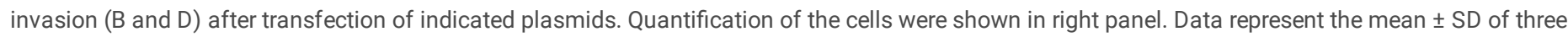
independent experiments. * $p<0.05$, ** $p<0.01$, *** $p<0.001$. 
A
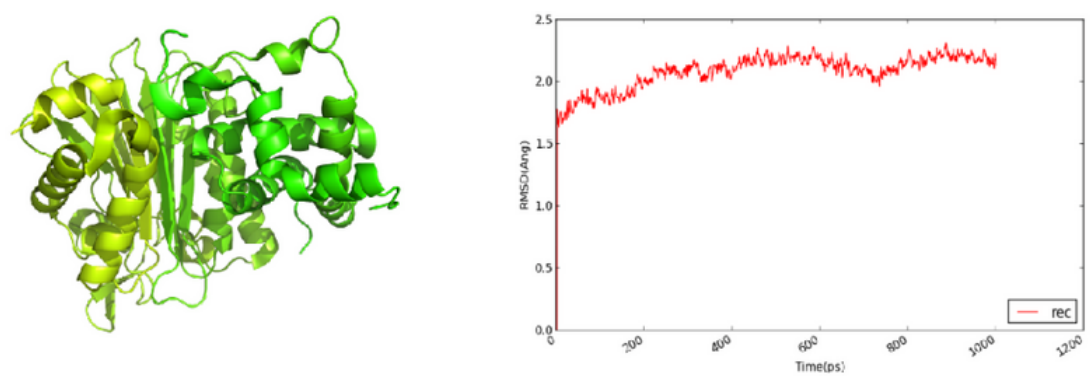

D
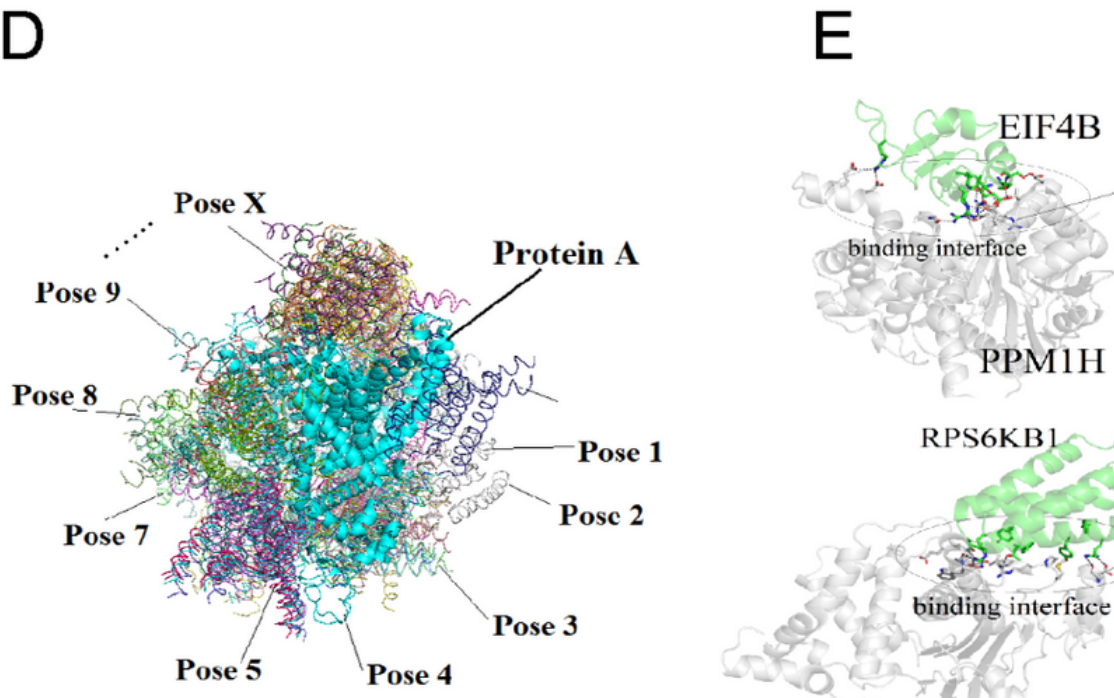

C

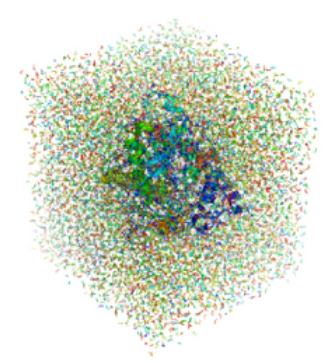

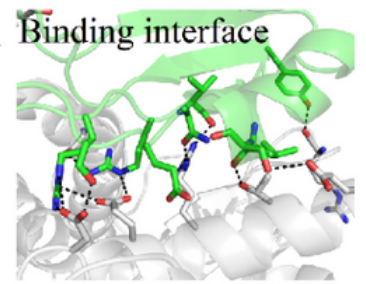
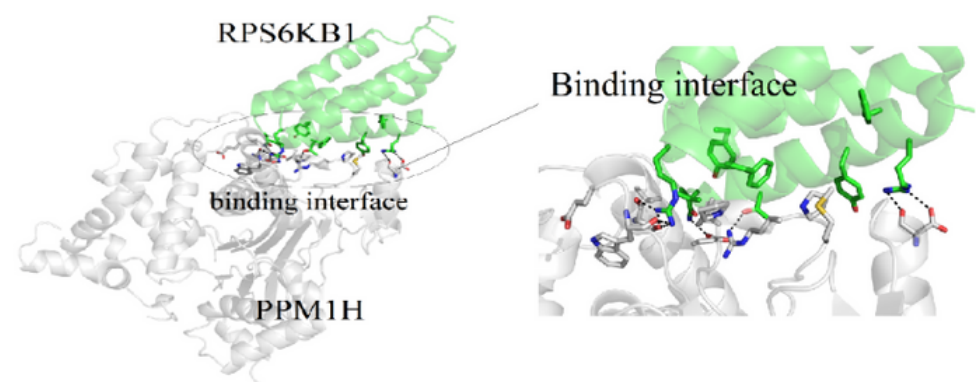

Figure 3

The computer simulates the PPM1H spatial simulation configuration and the docking mode of PPM1H with EIF4B and RPS6KB1. A: Computer simulation PPM1H spatial structure pattern diagram. B: RMSD value detection of the PPM1H spatial structure model. C: The Tip3P water box model analyzes the molecular dynamics trajectory of PPM1H. D: The optimal docking phase of PPM1H. E: Docking mode and the docking interface of PPM1H and EIF4B and RPS6KB1. 
A

HepG2

IP:Flag IP:Flag

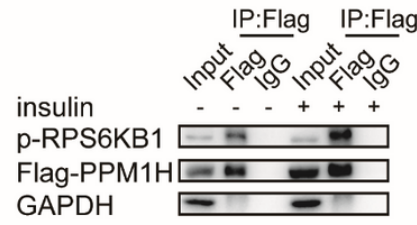

C

HepG2

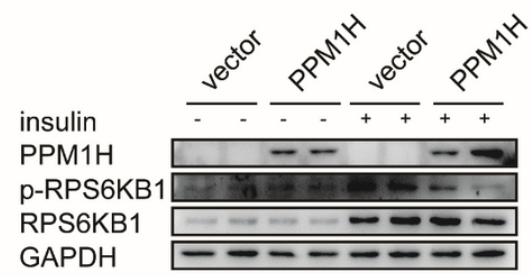

E

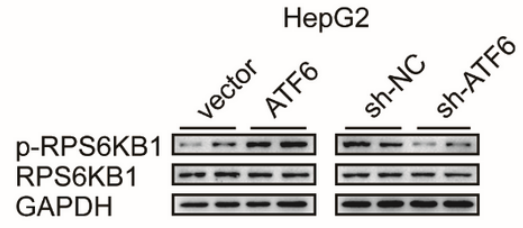

B

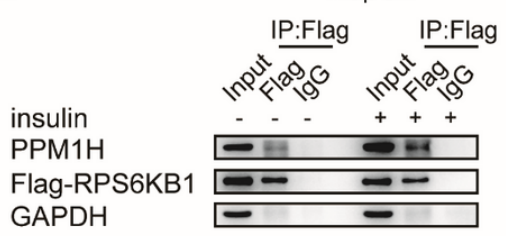

D

HepG2

His-PPM1H $(\mu \mathrm{g}) \quad 0 \quad 5 \quad 10 \quad 20$

PPM1H

p-RPS6KB1 -

RPS6KB1

$\mathrm{F}$

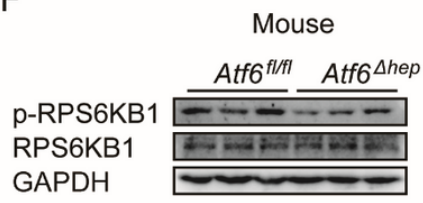

G

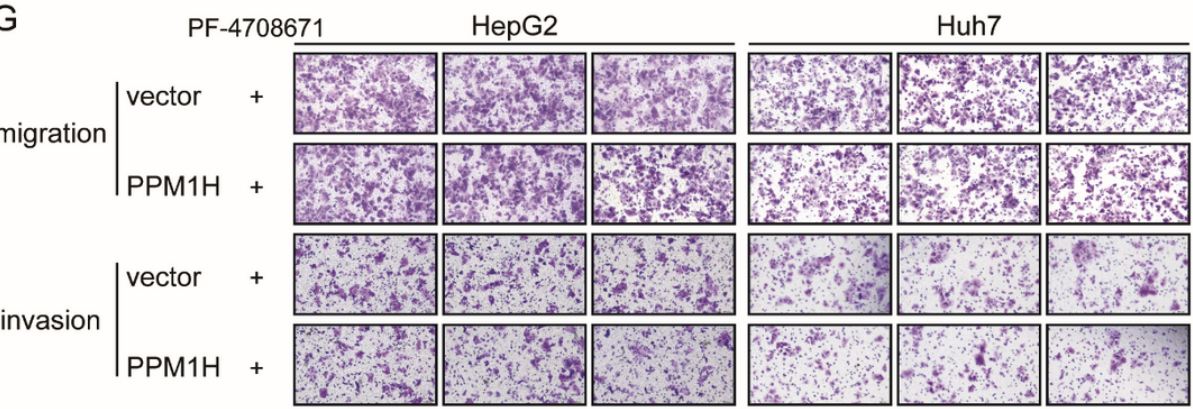

Figure 4

PPM1H directly dephosphorylated p-RPS6KB1. A-C: 24 hours past transfection, cells were cultured in basal medium without serum overnight, insulin(10ng/ $\mu$ l) were added 2 hours before harvest to active the phosphorylation of RPS6KB1. A: Flag-PPM1H were transfected into HepG2 cells with or without insulin treatment, and Co-IP were performed to examine the level of RPS6KB1 combined with PPM1H. B: Co-IP analysis of PPM1H and RPS6KB1 in Flag-RPS6KB1 transfected HepG2 cells with or without insulin treatment. C: Western blot analysis of the indicated protein level in PPM1 H-overexpressed HepG2 cells with or without insulin treatment. D: In vitro phosphatase assay to determine the interaction between PPM1H and p-RPS6KB1. E and F: Western blot analysis of the indicated protein level in vector, ATF6, sh-NC, sh-ATF6 transfected HepG2 cells (E), and in liver tissues of Atf6fl/fl or IAtf6 $\Delta$ hep mice. G: PF-4708671 is a RPS6KB1 inhibitor blocking the phosphorylation of RPS6KB1. It was added to the culture medium at a final concentration of $5 \mu \mathrm{M}$. Transwell assays revealed that the migration and invasion of PF-4708671-treated HepG2 and Huh7 cells showed little difference between vector and PPM1H. 
A

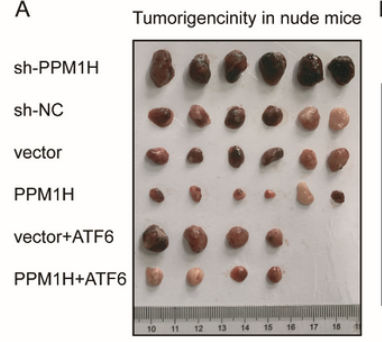

B

C

DEN/CCl4 induced mouse model
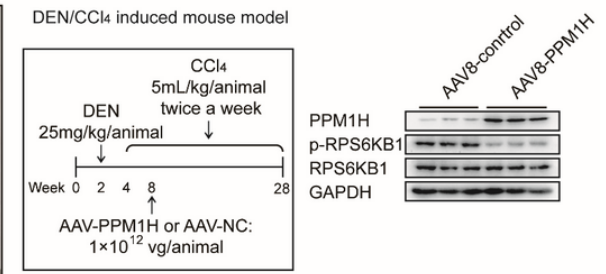

西

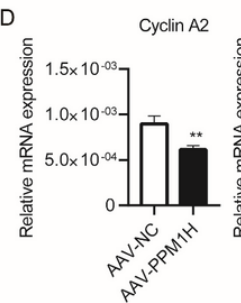

Cyclin B1 E

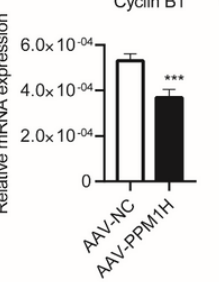

AAV8-NC

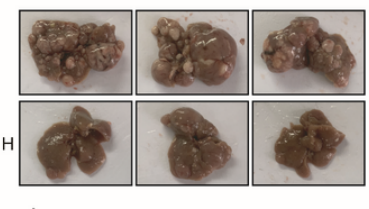

G

F

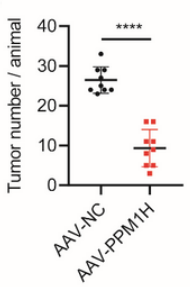

H

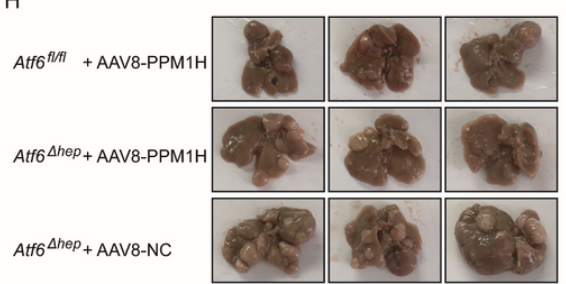

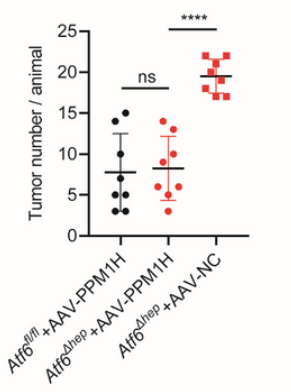

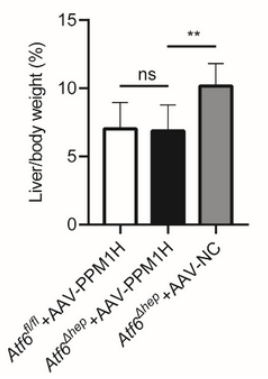

Figure 5

PPM1H suppressed tumor growth in mouse HCC models. A: Hep3B cells infected with indicating lentivirus were subcutaneously injected into male BALB/C nude mice, tumors were dissected out 4 weeks later. B: Scheme of the construction of DEN/CCl4-induced HCC mouse models. C and D: livers of wild type C57BL/ 6 mice were extracted 2 weeks after the injection of adeno-associated virus AAV8-control or AAV8-PPM1H to perform (C) western blot assay of PPM1H and p-RPS6KB1 protein level and (D) qRT-PCR analysis of cyclin A2 and cyclin B1 which are downstream genes of RPS6KB1, qRT-PCR data was normalized to GAPDH. E: Livers of DEN/CCl4-treated wild type C57BL/6 mice injected with AAV8-control or AAV8-PPM1H ( $\mathrm{n}=9$ per group). $\mathrm{F}$ and G: The tumor number and ratio of liver weight to body weight were measured. H: Livers of DEN/CCl4-treated Atf $6 \mathrm{fl} / \mathrm{fl}$ and Atf6 $\triangle$ hep mice injected with indicated AAV8 virus ( $\mathrm{n}=8 \mathrm{per}$ group). I and J: The tumor number and ratio of liver weight to body weight were measured. Data represent the mean $\pm S D$. ${ }^{*} p<0.05$, $* \star p<0.01$, $* \star \star p<0.001$,

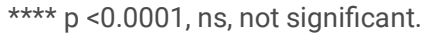


A

PPM1H

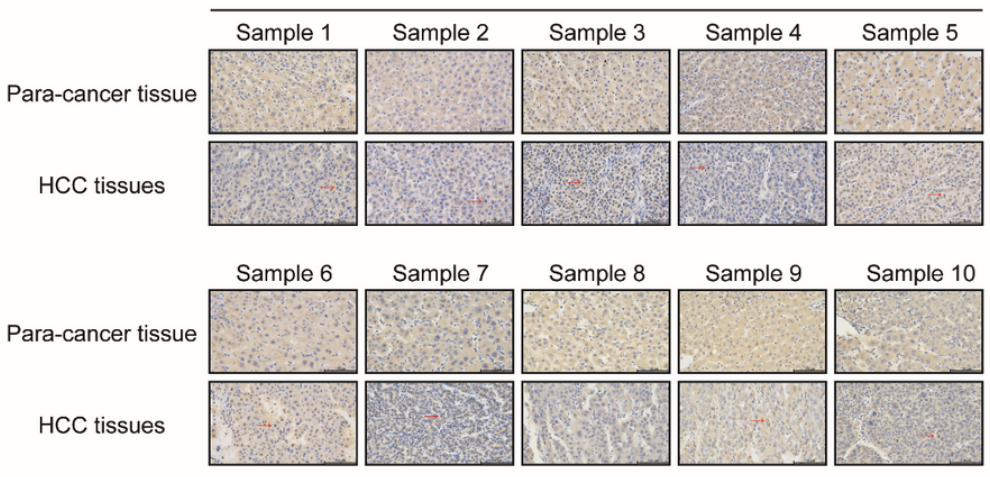

B

HCC tissues

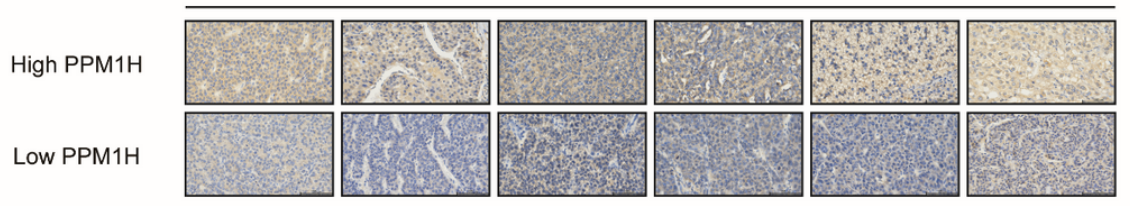

C

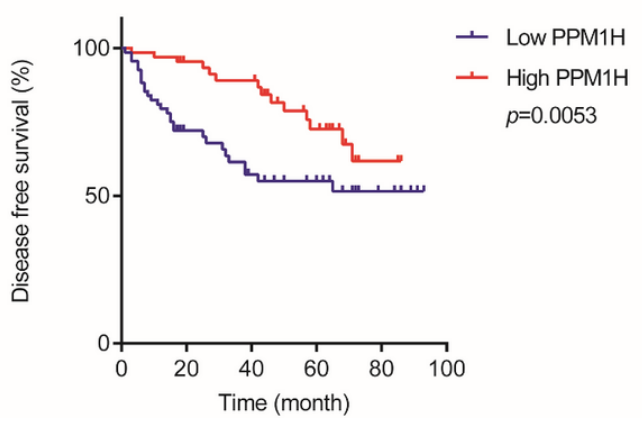

\section{Figure 6}

PPM1H predicted the progression of HCC patients. A: IHC analysis of human liver tissues. The red arrows indicated nuclear staining positive cells. B:

Representative IHC photos of human liver tissues with high or low PPM1H expression. C: Kaplan-Meier curves showed the survival of patients with low or high PPM1H expression. 


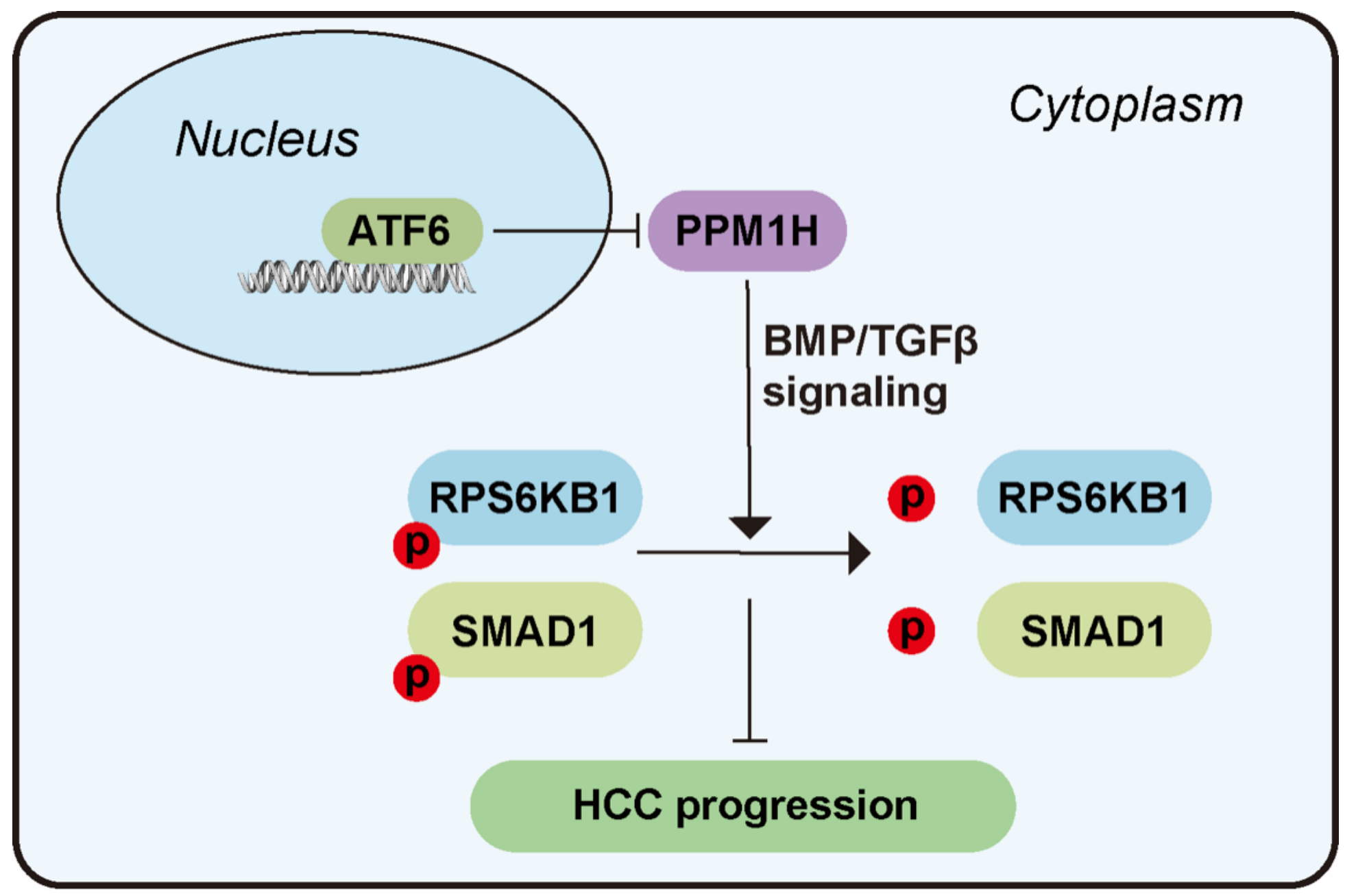

Figure 7

Schematic diagram of present study.

\section{Supplementary Files}

This is a list of supplementary files associated with this preprint. Click to download.

- Supplementalmaterial.docx 